\title{
INFINITE TIME INTERVAL BSDES AND THE CONVERGENCE OF $g$-MARTINGALES
}

\author{
ZENGJING CHEN and BO WANG
}

(Received 12 January 1999; revised 6 March 2000)

Communicated by V. T. Stefanov

\begin{abstract}
In this paper, we first give a sufficient condition on the coefficients of a class of infinite time interval backward stochastic differential equations (BSDEs) under which the infinite time interval BSDEs have a unique solution for any given square integrable terminal value, and then, using the infinite time interval BSDEs, we study the convergence of $g$-martingales introduced by Peng via a kind of BSDEs. Finally, we study the applications of $g$-expectations and $g$-martingales in both finance and economics.
\end{abstract}

2000 Mathematics subject classification: primary $60 \mathrm{H} 10,60 \mathrm{G} 48$.

Keywords and phrases: infinite time interval BSDEs, $g$-expectation, $g$-martingale, upcrossing inequality of $g$-martingale, convergence of $g$-martingale.

The adapted solution for a linear BSDE which appears as the adjoint process for a stochastic control problem was first introduced by Bismut in 1973, then by Bensoussan and others, while the first result for the existence and uniqueness of an adapted solution to a nonlinear BSDE with finite time interval and Lipschitzian coefficient was obtained by Pardoux and Peng [20]. Later many researchers developed the theory and its applications in a series of papers (see for example Darling [5], Hu and Peng [16], Lepeltier and Martin [18], Pardoux [19], Peng [24, 25], Karoui, Peng and Quenez [8] and the references therein) under some other assumptions on coefficients but for fixed terminal time. From these papers, the basic theorem is that, for a fixed terminal time $T>0$, under the suitable assumptions on terminal value $\xi$, coefficient $g$ and driving process $M$, the following BSDE has a solution pair $\left(y_{t}, z_{t}\right)$ in the interval $[0, T]$ :

$$
y_{t}=\xi+\int_{t}^{T} g\left(y_{s}, z_{s}, s\right) d s-\int_{t}^{T} z_{s} d M_{s}, \quad 0 \leq t \leq T .
$$

This work was partially supported by the National Natural Science Foundation of China, No. 79790130.

(c) 2000 Australian Mathematical Society 0263-6115/2000 \$A2.00+0.00 
More recently, Peng [25] introduced the notions of $g$-expectations and $g$-martingales via the above finite time interval BSDEs driven by a Brownian motion process. In Chen and Peng [1, 2] and Peng [24], some properties of $g$-martingales (such as upcrossing inequality, stopping sampling theorem and decomposition theorem for $g$ martingales) are discussed. As a supplement, in this paper, we discuss the convergence of $g$-martingales. One difficulty of this problem is how to study the existence and uniqueness of $\operatorname{BSDE}(0.1)$ when $T=\infty$. In fact, such a problem has been investigated by Peng [23], Pardoux [19], Darling and Pardoux [6], Pardoux and Zhang [21] and other researchers under the assumption that terminal value $\xi=0$ or $E e^{\rho T}|\xi|^{2}<\infty$ for some constant $\rho>0$ and random terminal time $T$. A natural question is under which conditions on $g$, does BSDE (0.1) still have a unique solution pair for any given square integrable $\xi$ when $T=\infty$ ? Obviously, the assumptions on $g$ in the papers mentioned above do not solve this question. In this paper, we first give a sufficient condition on coefficient $g$ under which for any square integrable random variable $\xi$, BSDE (0.1) still has a unique solution pair when $T=\infty$. Furthermore, we explain such a condition usually is necessary. We also give an example to show that our conditions on $g$ allow the coefficients to be unbounded, thus our result still extends Pardoux and Peng's result even for finite time horizon BSDE. Using these results, we show the convergence of $g$-martingales. Finally, we discuss some applications of $g$-expectations and $g$-martingales.

This paper is organized as follows. In Section 1, we consider a class of infinite time interval BSDEs: existence, uniqueness and convergence. In Section 2 we recall the notions of $g$-expectations and $g$-martingales introduced by Peng via BSDE. In Section 3 , we show the convergence of $g$-martingales. In Section 4 , we apply our results to economic theory and the pricing of contingent claims in incomplete security markets.

\section{Infinite time interval BSDEs}

Let $\left(\Omega, \mathscr{F}, \mathscr{F}_{t}, P\right)$ be a completed probability space, $\left(W_{t}\right)_{t \geq 0}$ be a $d$-dimensional standard Brownian motion. For the ease of exposition, we assume that the driving process $\left(M_{t}\right)$ in $\operatorname{BSDE}(0.1)$ is Brownian motion $\left(W_{t}\right)$ and $\left\{\mathscr{F}_{t}\right\}_{t \geq 0}$ is the natural filtration generated by Brownian motion $\left(W_{t}\right)_{t \geq 0}$, that is,

$$
\mathscr{F}_{t}=\sigma\left\{W_{s} ; s \leq t\right\} .
$$

Our method can be extended to the situation where $\left(W_{t}\right)$ is a martingale with jump and $\left\{\mathscr{F}_{t}\right\}$ is the usual filtration. Suppose $\mathscr{F}=\mathscr{F}_{\infty}=\sigma\left(\bigcup_{t \geq 0} \mathscr{F}_{t}\right)$, let us adopt the following notations.

$$
\begin{gathered}
S^{2}:=\left\{V: V \text { all }\left\{\mathscr{F}_{t}\right\}\right. \text {-adapted processes with norm of } \\
\left.\|V\|_{S^{2}}:=\left[E\left(\sup _{s \geq 0}|V(s)|\right)^{2}\right]^{1 / 2}<\infty\right\} ;
\end{gathered}
$$




$$
\begin{aligned}
\mathscr{M}^{2}(0, \infty):= & \left\{V: V \text { all }\left\{\mathscr{F}_{1}\right\}\right. \text {-adapted processes with norm of } \\
& \left.\|V\|_{M}:=\left[E \int_{0}^{\infty}|V(s)|^{2} d s\right]^{1 / 2}<\infty\right\} \\
L^{2}\left(\Omega, \mathscr{F}_{1}, P\right):= & \left\{\xi: \xi \text { all } \mathscr{F}_{t}\right. \text {-measurable random variables } \\
& \text { such that } \left.E|\xi|^{2}<\infty\right\}, \quad 0 \leq t \leq \infty \\
B^{2}:= & \left\{(X, Y): X \in S^{2}, Y \in \mathscr{M}^{2}(0, \infty)\right\} .
\end{aligned}
$$

For each $(X, Y) \in B^{2}$, we denote the norm of $(X, Y)$ by

$$
\|(X, Y)\|_{B^{2}}:=\left(\|X\|_{S^{2}}^{2}+\|Y\|_{M}^{2}\right)^{1 / 2} .
$$

Obviously, $B^{2}$ is a Banach space.

Consider the following infinite time interval BSDE

$$
y_{t}=\xi+\int_{t}^{\infty} g\left(y_{s}, z_{s}, s\right) d s-\int_{t}^{\infty} z_{s} d W_{s}
$$

where $\xi \in L^{2}(\Omega, \mathscr{F}, P)$ is given and $g \in \mathscr{L}(v, u)$. Here $\mathscr{L}(v, u)$ is the set of all functions $g: R \times R^{d} \times R_{+} \times \Omega \rightarrow R$ satisfying the following conditions:

(H1) For any $(y, z) \in R \times R^{d}, g(y, z, \cdot)$ is progressively measurable process such that $E\left(\int_{0}^{\infty}|g(y, z, s)| d s\right)^{2}<\infty$.

(H2) $g$ satisfies Lipschitz condition with Lipschitzian functions $v:=\{v(t)\}$ and $u:=\{u(t)\}$, that is, there exist two positive non-random functions $\{v(t)\}$ and $\{u(t)\}$ such that $\left|g\left(y_{1}, z_{1}, t\right)-g\left(y_{2}, z_{2}, t\right)\right| \leq v(t)\left|y_{1}-y_{2}\right|+u(t)\left|z_{1}-z_{2}\right|$, $\forall\left(y_{i}, z_{i}, t\right) \in R^{1+d} \times R_{+}, i=1,2$.

In this section, we prove that the following conditions on $v$ and $u$ solve our question:

$$
\text { (H3) } \int_{0}^{\infty} v(s) d s<\infty, \int_{0}^{\infty} u^{2}(s) d s<\infty
$$

To do so, let us first give an a priori estimate.

LEMMA 1.1. Suppose (H1), (H2) and (H3) hold for g. For any $T \in[0, \infty]$, let $Y_{T}^{i} \in L^{2}\left(\Omega, \mathscr{F}_{T}, P\right),\left(Y^{i}, Z^{i}\right)$ and $\left(y^{i}, z^{i}\right) \in B^{2}$ satisfy the following equations

(1.2) $Y_{t}^{i}=Y_{T}^{i}+\int_{t}^{T} g\left(y_{s}^{i}, z_{s}^{i}, s\right) d s-\int_{t}^{T} Z_{s}^{i} d W_{s}, \quad 0 \leq t \leq T \leq \infty ; \quad i=1,2$,

then for any $\tau \in[0, T]$,

$$
\begin{aligned}
& \left\|\left(\left(Y^{1}-Y^{2}\right) \mathbf{1}_{[\tau, T]},\left(Z^{1}-Z^{2}\right) \mathbf{1}_{[\tau, T]}\right)\right\|_{B^{2}}^{2} \\
& \quad \leq 20\left[E\left|Y_{T}^{1}-Y_{T}^{2}\right|^{2}+l(\tau, T)\left\|\left(\left(y^{1}-y^{2}\right) \mathbf{1}_{[\tau, T]},\left(z^{1}-z^{2}\right) \mathbf{1}_{[\tau, T]}\right)\right\|_{B^{2}}^{2}\right],
\end{aligned}
$$

where $l(\tau, T):=\left(\int_{\tau}^{T} v(s) d s\right)^{2}+\int_{\tau}^{T} u^{2}(s) d s$ and $\mathbf{1}_{[\tau, T]}(\cdot)$ is an indicator function. 
ProOF. Without loss of generality, we assume that $\tau=0, T=\infty$, otherwise we can replace $g$ by $g \mathbf{1}_{[r, T]}$. Set

$$
\begin{array}{ll}
\widehat{Y}:=Y^{1}-Y^{2}, & \widehat{Z}:=Z^{1}-Z^{2}, \quad \hat{y}:=y^{1}-y^{2}, \\
\hat{z}:=z^{1}-z^{2}, & \hat{g}_{t}:=g\left(y^{1}, z^{1}, t\right)-g\left(y^{2}, z^{2}, t\right) .
\end{array}
$$

Since $(\widehat{Y}, \widehat{Z}) \in B^{2},\left\{\int_{0}^{t} \widehat{Z}_{s} d W_{s}\right\}$ is a martingale and from (1.2) we have

$$
\widehat{Y}_{t}=E\left[\widehat{Y}_{T}+\int_{t}^{\infty} 1_{[r, T]}(s) \hat{g}_{s} d s / \mathscr{F}_{t}\right] \text {. }
$$

Applying Doob inequality, we deduce

$$
\begin{aligned}
\|\widehat{Y}\|_{S^{2}}^{2} & =E\left(\sup _{t \geq 0}\left|E\left[\widehat{Y}_{T}+\int_{t}^{\infty} 1_{(r, T]}(s) \hat{g}_{s} d s / \mathscr{F}_{t}\right]\right|\right)^{2} \\
& \leq E\left(\sup _{t \geq 0} E\left[\left|\widehat{Y}_{T}\right|+\int_{0}^{\infty}\left|\hat{g}_{s}\right| d s / \mathscr{F}_{t}\right]\right)^{2} \\
& \leq 4 E\left(\left|\widehat{Y}_{T}\right|+\int_{0}^{\infty}\left|\hat{g}_{s}\right| d s\right)^{2} \leq 8\left[E\left|\widehat{Y}_{T}\right|^{2}+E\left(\int_{0}^{\infty}\left|\hat{g}_{s}\right| d s\right)^{2}\right] .
\end{aligned}
$$

On the other hand, from (1.2) it follows that

$$
\begin{aligned}
\|\widehat{Z}\|_{M}^{2} & =E\left\langle\left.\int_{0} \hat{z}_{s} d W_{s}\right|_{\infty}\right. \\
& =E\left[\widehat{Y}_{T}+\int_{0}^{\infty} \hat{g}_{s} d s\right]^{2}-\left[E\left(\widehat{Y}_{T}+\int_{0}^{\infty} \hat{g}_{s} d s\right)\right]^{2} \\
& \leq E\left[\widehat{Y}_{T}+\int_{0}^{\infty} \hat{g}_{s} d s\right]^{2} \leq 2\left[E\left|\widehat{Y}_{T}\right|^{2}+E\left(\int_{0}^{\infty}\left|\hat{g}_{s}\right| d s\right)^{2}\right],
\end{aligned}
$$

where $\langle M\rangle$ is the variation process generated by the martingale $M$. Note that

$$
\begin{aligned}
E\left(\int_{0}^{\infty}\left|\hat{g}_{s}\right| d s\right)^{2} & \leq E\left[\int_{0}^{\infty}\left(v(s)\left|\hat{y}_{s}\right|+u(s)\left|\hat{z}_{s}\right|\right) d s\right]^{2} \\
& \leq 2 E\left[\int_{0}^{\infty} v(s) d s \cdot \sup _{t \geq 0}\left|\hat{y}_{t}\right|\right]^{2}+2 E\left[\int_{0}^{\infty} u^{2}(s) d s \cdot \int_{0}^{\infty}\left|\hat{z}_{s}\right|^{2} d s\right] \\
& =2\left[\left(\int_{0}^{\infty} v(s) d s\right)^{2} \cdot\|\hat{y}\|_{s^{2}}^{2}+\int_{0}^{\infty} u^{2}(s) d s \cdot\|\hat{z}\|_{M}^{2}\right] \\
& \leq 2\left[\left(\int_{0}^{\infty} v(s) d s\right)^{2}+\int_{0}^{\infty} u^{2}(s) d s\right]\|(\hat{y}, \hat{z})\|_{B^{2}}^{2}
\end{aligned}
$$




$$
=2 l(0, \infty)\|(\hat{y}, \hat{z})\|_{B^{2}}^{2} .
$$

Consequently, the last inequality, (1.3) and (1.4) imply

$$
\begin{aligned}
\|(\widehat{Y}, \widehat{Z})\|_{B^{2}}^{2}=\|\widehat{Y}\|_{S^{2}}^{2}+\|\widehat{Z}\|_{M}^{2} & \leq 10\left[E\left|\widehat{Y}_{T}\right|^{2}+E\left(\int_{0}^{\infty}\left|\hat{g}_{s}\right| d s\right)^{2}\right] \\
& \leq 20\left(E\left|\widehat{Y}_{T}\right|^{2}+l(0, \infty)\|(\hat{y}, \hat{z})\|_{B^{2}}^{2}\right) .
\end{aligned}
$$

For any $T, \tau \in[0, \infty]$ set $g_{1}(y, z, t):=g(y, z, t) \mathbf{1}_{[\tau, T]}$, then $g_{1}$ satisfies the assumption (H1), (H2) and (H3) and its Lipschitz constants are $v \mathbf{1}_{[\tau, T]}$ and $u \mathbf{1}_{[\tau, T]}$. Repeating the above process, we obtain the result.

THEOREM 1.2. Let $\xi \in L^{2}(\Omega, \mathscr{F}, P)$ be given, (H1), (H2) and (H3) hold for $g$, then BSDE (1.1) has a unique solution $(y, z) \in B^{2}$.

ProOF. We prove the theorem in two steps.

Step 1. We assume $\left[\left(\int_{0}^{\infty} v(s) d s\right)^{2}+\int_{0}^{\infty} u^{2}(s) d s\right]^{1 / 2}<1 / \sqrt{20}$. For any $(y, z) \in B^{2}$, applying (H1), (H2) and (H3) we derive

$$
\begin{aligned}
E\left(\xi+\int_{0}^{\infty} g\left(y_{s}, z_{s}, s\right) d s\right)^{2} \leq & E\left(|\xi|+\int_{0}^{\infty}\left|g\left(y_{s}, z_{s}, s\right)\right| d s\right)^{2} \\
\leq & E\left[|\xi|+\int_{0}^{\infty}\left(|g(0,0, s)|+v(s)\left|y_{s}\right|+u(s)\left|z_{s}\right|\right) d s\right]^{2} \\
\leq & 4 E \xi^{2}+4 E\left(\int_{0}^{\infty}(g(0,0, s) d s)^{2}\right. \\
& +4 E\left(\int_{0}^{\infty} v(s)\left|y_{s}\right| d s\right)^{2}+4 E\left(\int_{0}^{\infty} u(s)\left|z_{s}\right| d s\right)^{2}
\end{aligned}
$$

Since

$$
E\left[\int_{0}^{\infty} v(s)\left|y_{s}\right| d s\right]^{2} \leq E\left[\int_{0}^{\infty} v(s) d s \cdot \sup _{t \geq 0}\left|y_{t}\right|\right]^{2}=\left(\int_{0}^{\infty} v(s) d s\right)^{2}\|y\|_{S^{2}}^{2}<\infty
$$

and

$$
E\left[\int_{0}^{\infty} u(s)\left|z_{s}\right| d s\right]^{2} \leq E\left[\int_{0}^{\infty} u^{2}(s) d s \cdot \int_{0}^{\infty}\left|z_{s}\right|^{2} d s\right]=\int_{0}^{\infty} u^{2}(s) d s \cdot\|z\|_{M}^{2}<\infty,
$$

we have

$$
E\left[\xi+\int_{0}^{\infty} g\left(y_{s}, z_{s}, s\right) d s\right]^{2}<\infty
$$


which means $\left\{E\left[\xi+\int_{0}^{\infty} g\left(y_{s}, z_{s}, s\right) d s / \mathscr{F}_{t}\right]\right\}_{t \geq 0}$ is a square integrable martingale. According to the martingale representation theorem, there exists a unique $Z \in \mathscr{M}^{2}(0, \infty)$ such that

Let

$$
\begin{aligned}
E\left[\xi+\int_{0}^{\infty} g\left(y_{s}, z_{s}, s\right) d s / \mathscr{F}_{1}\right]= & E\left[\xi+\int_{0}^{\infty} g\left(y_{s}, z_{s}, s\right) d s\right] \\
& +\int_{0}^{t} Z_{s} d W_{s}, \quad 0 \leq t \leq \infty .
\end{aligned}
$$

$$
Y_{t}:=E\left[\xi+\int_{t}^{\infty} g\left(y_{s}, z_{s}, s\right) d s / \mathscr{F}_{t}\right], \quad 0 \leq t \leq \infty .
$$

Obviously, $(Y, Z) \in B^{2}$. Equations (1.5) and (1.6) have constructed a mapping from $B^{2}$ to $B^{2}$, and we denote it by $\phi$, that is,

$$
\phi:(y, z) \rightarrow(Y, Z) .
$$

If $\phi$ is a contractive mapping with respect to the norm $\|\cdot\|_{B^{2}}$, by the fixed point theorem, there exists a unique $(y, z) \in B^{2}$ satisfying (1.5) and (1.6), that is,

$$
\left\{\begin{array}{l}
E\left[\xi+\int_{0}^{\infty} g\left(y_{s}, z_{s}, s\right) d s / \mathscr{F}_{t}\right]=E\left(\xi+\int_{0}^{\infty} g\left(y_{s}, z_{s}, s\right) d s\right)+\int_{0}^{t} z_{s} d W_{s} ; \\
y_{t}=E\left[\xi+\int_{t}^{\infty} g\left(y_{s}, z_{s}, s\right) d s / \mathscr{F}_{t}\right], \quad 0 \leq t \leq \infty,
\end{array}\right.
$$

which is equivalent to BSDE (1.1).

We now prove that $\phi$ is a contractive mapping. Suppose $\left(y^{i}, z^{i}\right) \in B^{2}$, let $\left(Y^{i}, Z^{i}\right)$ be the map of $\left(y^{i}, z^{i}\right),(i=1,2)$, that is,

$$
\phi\left(y^{i}, z^{i}\right)=\left(Y^{i}, Z^{i}\right), \quad i=1,2 .
$$

We denote

$$
\begin{array}{ll}
\widehat{Y}:=Y^{1}-Y^{2}, & \widehat{Z}:=Z^{1}-Z^{2}, \quad \hat{y}:=y^{1}-y^{2}, \\
\hat{z}:=z^{1}-z^{2}, & \hat{g}_{t}:=g\left(y^{1}, z^{1}, t\right)-g\left(y^{2}, z^{2}, t\right) .
\end{array}
$$

By Lemma 1.1 we have

$$
\begin{aligned}
\left\|\phi\left(y^{1}, z^{1}\right)-\phi\left(y^{2}, z^{2}\right)\right\|_{B^{2}} & =\|(\widehat{Y}, \widehat{Z})\|_{B^{2}} \\
& \leq \sqrt{20}\left[\left(\int_{0}^{\infty} v(s) d s\right)^{2}+\int_{0}^{\infty} u^{2}(s) d s\right]^{1 / 2}\|(\hat{y}, \hat{z})\|_{B^{2}} .
\end{aligned}
$$

Note that $\left[\left(\int_{0}^{\infty} v(s) d s\right)^{2}+\int_{0}^{\infty} u^{2}(s) d s\right]^{1 / 2}<1 / \sqrt{20}$. Thus $\phi$ is a contractive mapping from $B^{2}$ to $B^{2}$. 
Step 2. Since $\int_{0}^{\infty} v(s) d s<\infty, \int_{0}^{\infty} u^{2}(s) d s<\infty$, then there exists a sufficiently large constant $T$ such that

$$
\left[\left(\int_{T}^{\infty} v(s) d s\right)^{2}+\int_{T}^{\infty} u^{2}(s) d s\right]^{1 / 2}<\frac{1}{\sqrt{20}}
$$

Let $g_{1}(y, z, t):=\mathbf{1}_{[T, \infty]}(t) g(y, z, t)$, then (H1), (H2) and (H3) hold on $g_{1}$ whose Lipschitzian constants are $\bar{v}(t)=\mathbf{1}_{[T, \infty]}(t) v(t)$ and $\bar{u}(t)=\mathbf{1}_{[T, \infty]}(t) u(t)$. Obviously,

$$
\left[\left(\int_{0}^{\infty} \bar{v}(s) d s\right)^{2}+\int_{0}^{\infty} \bar{u}^{2}(s) d s\right]^{1 / 2}<\frac{1}{\sqrt{20}}
$$

By Step 1 , there exists a unique $(\hat{y}, \hat{z}) \in B^{2}$ such that

$$
\hat{y}_{t}=\xi+\int_{t}^{\infty} g_{1}\left(\hat{y}_{s}, \hat{z}_{s}, s\right) d s-\int_{t}^{\infty} \hat{z}_{s} d W_{s}, \quad 0 \leq t \leq \infty .
$$

For $\left(\hat{y}_{t}, \hat{z}_{t}\right)$ given as above, let us consider the following infinite BSDE:

$$
\left\{\begin{array}{l}
\bar{y}_{t}=\int_{t}^{T} g\left(\bar{y}_{s}+\hat{y}_{s}, \bar{z}_{s}+\hat{z}_{s}, s\right) d s-\int_{t}^{T} \bar{z}_{s} d W_{s}, \quad 0 \leq t \leq T ; \\
\bar{y}_{t} \equiv 0, \quad \bar{z}_{t} \equiv 0, \quad t>T .
\end{array}\right.
$$

According to the Pardoux-Peng theorem (see Pardoux and Peng [20]), the above BSDE has a unique solution $\left(\bar{y}_{t}, \bar{z}_{t}\right)$ in $[0, T]$, thus the above BSDE has a unique solution such that $\left(\bar{y}_{t}, \bar{z}_{t}\right)=(0,0)$, for every $t \geq T$.

Let

$$
y:=\bar{y}+\hat{y}, \quad z:=\bar{z}+\hat{z} .
$$

It is easy to check that $\left(y_{t}, z_{t}\right)$ is the unique solution of BSDE (1.1).

REMARK 1. Suppose $\mu$ is a constant, if we choose $v(t)=u(t):=\mu \mathbf{1}_{[0, T]}(t)$, then Theorem 1.1 is the main theorem in Pardoux and Peng [20].

REMARK 2. The condition (H3) usually is necessary. That is, if for any $\xi$ and $g \in \mathscr{L}(v, u)$, BSDE (1.1) has a unique solution in $B^{2}$, then the assumption (H3) is necessary.

In fact, let us choose $g(y, z, t)=v(t) y$. Obviously, $g \in \mathscr{L}(v, u)$ and for any $\xi:=a,(a>0$ is a constant $)$, the solution of BSDE

$$
y_{t}=a+\int_{t}^{\infty} v(s) y_{s} d s-\int_{t}^{\infty} z_{s} d W_{s}, \quad 0 \leq t \leq \infty
$$


is $y_{t}=a \exp \left(\int_{t}^{\infty} v(s) d s\right)$, and $z_{t}=0$. Since $y_{0}<\infty$, then $\int_{0}^{\infty} v(s) d s<\infty$.

Similarly, if we choose $g(y, z, t)=u(t) z$, then the solution of BSDE

should be

$$
y_{t}=\xi+\int_{t}^{\infty} u(s) z_{s} d s-\int_{t}^{\infty} z_{s} d W_{s}
$$

$$
y_{t}=E\left[\xi \exp \left(-\frac{1}{2} \int_{t}^{\infty} u^{2}(s) d s+\int_{t}^{\infty} u(s) d W_{s}\right) \mid \mathscr{F}_{t}\right]
$$

Thus the assumption $\int_{0}^{\infty} u^{2}(s) d s<\infty$ is necessary.

The following example shows that our assumptions allow the coefficients of BSDE (1.1) to be unbounded. Thus Theorem 1.2 generalizes the result in Pardoux and Peng [20] even for finite time horizon BSDE.

EXAMPLE 1. For given $T>0$, let $\xi \in L^{2}\left(\Omega, \mathscr{F}_{T}, P\right)$, then the following BSDE has a unique solution:

$$
y_{t}=\xi+\int_{t}^{T}\left[\frac{1}{(T-s)^{1 / 2}}\left|y_{s}\right|+\frac{1}{(T-s)^{1 / 4}}\left|z_{s}\right|\right] d s-\int_{t}^{T} z_{s} d W_{s}, \quad 0 \leq t \leq T .
$$

In fact, let us choose $v$ and $u$ as follows:

$$
v(t):=\left\{\begin{array}{ll}
(T-t)^{-1 / 2}, & 0 \leq t<T ; \\
0, & t \geq T ;
\end{array} \quad u(t):= \begin{cases}(T-t)^{-1 / 4}, & 0 \leq t<T ; \\
0, & t \geq T .\end{cases}\right.
$$

It is easy to show that $v$ and $u$ satisfy condition (H3), but $v$ and $u$ are unbounded.

We now discuss the convergence of the solutions of infinite time interval BSDEs. The following theorem is called the Continuous Dependence Theorem.

THEOREM 1.3. Suppose $\xi_{i} \in L^{2}(\Omega, \mathscr{F}, P),(i=1,2)$, let $\left(y^{i}, z^{i}\right)$ be the solutions of BSDE (1.1) corresponding to $\xi=\xi_{1}, \xi=\xi_{2}$, respectively, then there exists a constant $C>0$ such that

$$
\left\|\left(y^{1}-y^{2}, z^{1}-z^{2}\right)\right\|_{B^{2}}^{2} \leq C E\left|\xi_{1}-\xi_{2}\right|^{2} .
$$

PROOF. Set $\hat{y}:=y^{1}-y^{2} ; \hat{z}:=z^{1}-z^{2}$. Since $\left(\int_{0}^{\infty} v(s) d s\right)^{2}+\int_{0}^{\infty} u^{2}(s) d s<\infty$, we can choose a strictly increasing sequence $0=t_{0}<t_{1}<\cdots<t_{n}<t_{n+1}=\infty$ such that

$$
a_{i}:=\left[\left(\int_{t_{i}}^{t_{i+1}} v(s) d s\right)^{2}+\int_{t_{i}}^{t_{i+1}} u^{2}(s) d s\right]^{1 / 2}<\frac{1}{\sqrt{20}}, \quad i=0,1, \ldots, n .
$$


Set $L:=\max _{i} a_{i}$, since $1-20 L^{2}>0$, thus $N:=\sqrt{20} / \sqrt{1-20 L^{2}}$ is well defined and $N>1$. Applying Lemma 1.1 we have

$$
\begin{aligned}
\left\|(\hat{y}, \hat{z}) \mathbf{1}_{\left[t_{i}, t_{i+1}\right]}\right\|_{B^{2}}^{2} & \leq 20 E \hat{y}_{t_{i+1}}^{2}+20\left[\left(\int_{t_{i}}^{t_{i+1}} v(s) d s\right)^{2}+\int_{t_{i}}^{t_{i+1}} u^{2}(s) d s\right]\left\|(\hat{y}, \hat{z}) \mathbf{1}_{\left[t_{i}, t_{i+1}\right]}\right\|_{B^{2}}^{2} \\
& \leq 20 E \hat{y}_{t_{i+1}}^{2}+20 L^{2}\left\|(\hat{y}, \hat{z}) \mathbf{1}_{\left[t_{i}, t_{i+1}\right]}\right\|_{B^{2}}^{2} .
\end{aligned}
$$

Thus

$$
\begin{aligned}
\left\|(\hat{y}, \hat{z}) \mathbf{1}_{\left[t_{i}, t_{i+1}\right]}\right\|_{B^{2}}^{2} & \leq \frac{20}{1-20 L^{2}} E \hat{y}_{t_{i+1}}^{2}=N^{2} E \hat{y}_{t_{i+1}}^{2} \\
& \leq N^{2} E\left[\left(\sup _{t_{i+1} \leq s \leq t_{i+2}}\left|\hat{y}_{s}\right|\right)^{2}+\int_{t_{i}}^{t_{i+2}} \hat{z}_{s}^{2} d s\right] \\
& =N^{2}\left\|(\hat{y}, \hat{z}) \mathbf{1}_{\left[t_{i+1}, t_{i+2}\right]}\right\|_{B^{2}}^{2}, \quad i=0,1, \ldots, n-1 .
\end{aligned}
$$

In particular,

$$
\left\|(\hat{y}, \hat{z}) \mathbf{1}_{\left[t_{n}, \infty\right]}\right\|_{B^{2}}^{2} \leq N^{2} E\left|\xi_{1}-\xi_{2}\right|^{2} .
$$

From (1.7) and (1.8) we have

$$
\begin{aligned}
\|(\hat{y}, \hat{z})\|_{B^{2}} & \leq \sum_{i=0}^{n}\left\|(\hat{y}, \hat{z}) \mathbf{1}_{\left[t_{i}, t_{i+1}\right]}\right\|_{B^{2}} \leq\left(N^{n+1}+\cdots+N\right)\left[E\left(\xi_{1}-\xi_{2}\right)^{2}\right]^{1 / 2} \\
& =\frac{N\left(N^{n+1}-1\right)}{N-1}\left(E\left|\xi_{1}-\xi_{2}\right|^{2}\right)^{1 / 2} .
\end{aligned}
$$

Thus $\|(\hat{y}, \hat{z})\|_{B^{2}}^{2} \leq C E\left|\xi_{1}-\xi_{2}\right|^{2}$.

Using Theorem 1.3, we can obtain the following convergence theorem for infinite time interval BSDE.

THEOREM 1.4. Suppose $\xi, \xi_{k} \in L^{2}(\Omega, \mathscr{F}, P),(k=1,2, \ldots)$, (H1), (H2) and (H3) hold for $g$. Let $\left(y^{k}, z^{k}\right)$ be the solution of the following BSDEs:

$$
y_{t}^{k}=\xi_{k}+\int_{t}^{\infty} g\left(y_{s}^{k}, z_{s}^{k}, s\right) d s-\int_{t}^{\infty} z_{s}^{k} d W_{s}, \quad k=1,2, \ldots
$$

If $E\left|\xi_{k}-\xi\right|^{2} \rightarrow 0$ as $k \rightarrow \infty$, then there exists a pair $(y, z) \in B^{2}$ such that $\left\|\left(y^{k}-y, z^{k}-z\right)\right\|_{B^{2}} \rightarrow 0$ as $k \rightarrow \infty$. Furthermore, $(y, z)$ is the solution of the following BSDE:

$$
y_{t}=\xi+\int_{t}^{\infty} g\left(y_{s}, z_{s}, s\right) d s-\int_{t}^{\infty} z_{s} d W_{s}
$$


PROOF. For any $n, m>1$, let $\left(y^{n}, z^{n}\right)$ and $\left(y^{m}, z^{m}\right)$ be the solutions of BSDE (1.9) corresponding to $\xi_{k}=\xi_{n}$ and $\xi_{k}=\xi_{m}$. Due to Theorem 1.3, there exists a constant $C$ such that

$$
\begin{aligned}
\left\|\left(y^{n}-y^{m}, z^{n}-z^{m}\right)\right\|_{B^{2}}^{2} & \leq C E\left|\xi_{n}-\xi_{m}\right|^{2} \\
& \leq 2 C\left[E\left|\xi_{n}-\xi\right|^{2}+E\left|\xi_{m}-\xi\right|^{2}\right] \rightarrow 0 \quad \text { as } n, m \rightarrow \infty
\end{aligned}
$$

which means that $\left(y^{n}, z^{n}\right)$ is a Cauchy sequence in $B^{2}$, Thus there exists a pair $(y, z) \in B^{2}$ such that $\left\|\left(y^{n}-y, z^{n}-z\right)\right\|_{B^{2}} \rightarrow 0$ as $n \rightarrow \infty$.

Since

$$
\begin{aligned}
& E \int_{0}^{\infty}\left|g\left(y_{z}^{n}, z_{s}^{n}, s\right)-g\left(y_{s}, z_{s}, s\right)\right| d s \\
& \quad \leq E \int_{0}^{\infty}\left[v(s)\left|y_{z}^{n}-y_{s}\right|+u(s)\left|z_{s}^{n}-z_{s}\right|\right] d s \\
& \quad \leq 2\left[\left(\int_{0}^{\infty} v(s) d s\right)^{2}+\int_{0}^{\infty} u^{2}(s) d s\right]^{1 / 2}\left\|\left(y^{n}-y, z^{n}-z\right)\right\|_{B^{2}} \rightarrow 0 \text { as } n \rightarrow \infty .
\end{aligned}
$$

Thus $\int_{0}^{\infty} g\left(y_{s}^{n}, z_{s}^{n}\right) d s \rightarrow \int_{0}^{\infty} g\left(y_{s}, z_{s}, s\right) d s$ as $n \rightarrow \infty$ in $L^{1}(\Omega, \mathscr{F}, P)$. Taking the limit on both sides of $\operatorname{BSDE}(1.9)$, we deduce that $(y, z)$ is the solution of $\operatorname{BSDE}(1.10)$.

The following corollary shows the relation of solutions between infinite time interval BSDE (1.10) and the following finite time interval BSDE:

$$
y_{t}=E\left[\xi / \mathscr{F}_{T}\right]+\int_{t}^{T} g\left(y_{s}, z_{s}, s\right) d s-\int_{t}^{T} z_{s} d W_{s}, \quad 0 \leq t \leq T<\infty .
$$

COROllaRY 1.5. Assume $\xi \in L^{2}(\Omega, \mathscr{F}, P)$, (H1), (H2) and (H3) hold for g. Let $(y, z)$ be the solution of BSDE (1.10). For any $T>0$, let $\left(y^{T}, z^{T}\right)$ be the solution of finite time interval BSDE (1.11), then $\left(y^{T}, Z^{T}\right) \rightarrow(y, z)$ as $T \rightarrow \infty$ in $B^{2}$.

ProOF. Note that $E\left[\xi / \mathscr{F}_{T}\right] \rightarrow \xi$ in $L^{2}(\Omega, \mathscr{F}, P)$ as $T \rightarrow \infty$. The proof is straightforward from Theorem 1.4.

\section{2. $g$-expectation, $g$-martingale}

Peng [25] introduced the notions of $g$-expectations and $g$-martingales via a finite time interval BSDE under the following assumption:

(H4) $g(y, 0, t) \equiv 0$, for every $(y, t) \in R \times R_{+}$. 
In this section, we first present the general notions of g-expectations and the corresponding $g$-martingales via the infinite time interval BSDE introduced in Section 1 under the hypothesis (H4).

Definition 2.1. Suppose $\xi \in L^{2}(\Omega, \mathscr{F}, P)$ is given, (H1), (H2), (H3) and (H4) hold for $g$. Let $\left(y_{t}, z_{t}\right) \in B^{2}$ be the solution of BSDE (1.1). We call $\mathscr{E}_{g}[\xi]$, where

$$
\mathscr{E}_{g}[\xi]:=y_{0}
$$

the g-expectation of random variable $\xi$.

In particular, if $g(y, z, t)= \pm u(t)|z|$, we rewrite $\mathscr{E}_{g}[\xi]$ simply as $\mathscr{E}_{ \pm u}[\xi]$, where $u$ is Lipschitz function defined in (H2) and (H3).

LEMMA 2.2. Suppose $\xi \in L^{2}(\Omega, \mathscr{F}, P)$ and $(\mathrm{H} 1)-(\mathrm{H} 4)$ hold for $g$, let $\left(y_{t}^{\xi}, z_{t}^{\xi}\right)$ be the solution of $\mathrm{BSDE}$ (1.1). Then for any $A \in \mathscr{F}_{r}(0 \leq r \leq \infty)$ we have

$$
y_{r}^{\mathbf{1}_{A} \xi}=\mathbf{1}_{A} y_{r}^{\xi}, \quad r \geq 0 .
$$

Here $\left\{y_{t}^{\mathbf{1}^{\xi} \xi}\right\}$ is the solution of BSDE (1.1) corresponding to the terminal value $\mathbf{1}_{A} \xi$.

PROOF. Set $\left(y_{t}, z_{t}\right):=\left(y_{t}^{\xi}, z_{t}^{\xi}\right)$, for any $A \in \mathscr{F}_{r}$, multiplying $\mathbf{1}_{A}$ on both sides of $\operatorname{BSDE}(1.1)$, we have

$$
\mathbf{1}_{A} y_{t}=\mathbf{1}_{A} \xi+\int_{t}^{\infty} \mathbf{1}_{A} g\left(y_{s}, z_{s}, s\right) d s-\int_{t}^{\infty} \mathbf{1}_{A} z_{s} d W_{s}, \quad 0 \leq t \leq \infty
$$

Since $y_{t} \mathbf{1}_{A}$ is $\mathscr{F}_{t}$-measurable for each $t \in[r, \infty]$ and the relation $g\left(\mathbf{1}_{A} y, z \mathbf{1}_{A}, t\right)=$ $\mathbf{1}_{A} g(y, z, t)$, for every $(y, z, t) \in \mathbb{R}^{1+d} \times R_{+}$holds under the assumption (H4), we obtain that $\left(y_{t} \mathbf{1}_{A}, z_{t} \mathbf{1}_{A}\right)$ solves the following BSDE

$$
\bar{y}_{t}=\mathbf{1}_{A} \xi+\int_{t}^{\infty} g\left(\bar{y}_{s}, \bar{z}_{s}, s\right) d s-\int_{t}^{\infty} \bar{z}_{s} d W_{s}, \quad 0 \leq t \leq \infty,
$$

when $r \leq t \leq \infty$.

It then follows, by the uniqueness of the solution of BSDE (2.1), that

$$
\mathbf{1}_{A} y_{t}=\bar{y}_{t}, \quad t \in[r, \infty]
$$

which means that $\mathbf{1}_{A} y_{t}^{\xi}=y_{t}^{\mathbf{1}_{A} \xi}$ for every $t \in[r, \infty]$. In particular, $\mathbf{1}_{A} y_{r}^{\xi}=y_{r}^{\mathbf{1}_{A} \xi}$. The proof is complete.

The following lemma follows directly from the assumption (H4). 
LEMMA 2.3. Suppose $\xi \in L^{2}\left(\Omega, \mathscr{F}_{r}, P\right),(r \geq 0)$, (H1)-(H4) hold for $g$. Let $\left(y_{t}, z_{t}\right)$ be the solution of infinite time interval BSDE (1.1) and let $\left(\bar{y}_{t}, \bar{z}_{t}\right)$ be the solution of the following finite time interval BSDE

$$
\bar{y}_{t}=\xi+\int_{t}^{r} g\left(\bar{y}_{s}, \bar{z}_{s}, s\right) d s-\int_{t}^{r} \bar{z}_{s} d W_{s}, \quad 0 \leq t \leq r .
$$

Then

$$
y_{t}=\bar{y}_{t}, \quad \forall t \in[0, r]
$$

In particular, $y_{0}=\bar{y}_{0}$.

PROOF. It is easy to check that $\left(\bar{y}_{t} \mathbf{1}_{[0, r](t)}+\xi \mathbf{1}_{(r, \infty]}, \bar{z}_{t} \mathbf{1}_{[0, r]}\right)$ is the solution of BSDE (1.1). It then follows, by the uniqueness of $\operatorname{BSDE}(1.1)$, that we obtain the following fact:

$$
y_{t}=\left\{\begin{array}{ll}
\bar{y}_{t}, & t \leq r ; \\
\xi, & t>r,
\end{array} \quad z_{t}= \begin{cases}\bar{z}_{t}, & t \leq r \\
0, & t>r .\end{cases}\right.
$$

In particular, $y_{0}=\bar{y}_{0}$.

LEMMA 2.4. Suppose $\xi \in L^{2}(\Omega, \mathscr{F}, P)$, then there exists a unique random variable $\eta \in L^{2}\left(\Omega, \mathscr{F}_{t}, P\right)$, such that

$$
\mathscr{E}_{g}\left[\mathbf{1}_{A} \xi\right]=\mathscr{E}_{g}\left[\mathbf{1}_{A} \eta\right], \quad \forall A \in \mathscr{F}_{t}, \quad 0 \leq t \leq \infty .
$$

(We call $\eta$ the conditional expectation of random variable $\xi$ generated by function $g$ under $\mathscr{F}_{t}$ and write it as $\mathscr{E}_{g}\left[\xi / \mathscr{F}_{t}\right]$.) Furthermore,

$$
\mathscr{E}_{g}\left[\xi / \mathscr{F}_{t}\right]=y_{t}^{\xi}
$$

where $y_{t}^{\xi}$ is the value of the solution $\left\{y_{t}^{\xi}\right\}$ of BSDE (1.1) at time $t$.

Proof. For any $A \in \mathscr{F}_{t}$, by the definition of $\mathscr{E}_{g}[\cdot]$ and Lemma 2.2, we have

$$
\mathscr{E}_{g}\left[\mathbf{1}_{A} \xi\right]=\mathscr{E}_{g}\left[y_{t}^{\mathbf{1}_{A} \xi}\right]=\mathscr{E}_{g}\left[\mathbf{1}_{A} y_{t}^{\xi}\right]
$$

Set $\eta:=y_{t}^{\xi}$, then $\eta \in L^{2}\left(\Omega, \mathscr{F}_{t}, P\right)$.

We now prove that $\eta$ is unique. Indeed, if there exists another $\bar{\eta} \in L^{2}\left(\Omega, \mathscr{F}_{t}, P\right)$ such that

$$
\mathscr{E}_{g}\left[\mathbf{1}_{A} \eta\right]=\mathscr{E}_{g}\left[\mathbf{1}_{A} \bar{\eta}\right], \quad \forall A \in \mathscr{F}_{t}
$$

but $P(\eta \neq \bar{\eta})>0$. 
Set $B:=\{\eta>\bar{\eta}\}$, without loss of generality, we assume that $P(B)>0$. It then follows, by Theorem A.3(2)(i) in Appendix, that

$$
\mathscr{E}_{g}\left[\mathbf{1}_{B} \eta\right]>\mathscr{E}_{g}\left[\mathbf{1}_{B} \bar{\eta}\right],
$$

which is contrary to equality (2.3).

REMARK 3. The notation $\mathscr{E}_{g}[\xi]$ and $\mathscr{E}_{g}\left[\cdot / \mathscr{F}_{l}\right]$ comes from Peng [25]. Obviously, if $g \equiv 0$, then $\mathscr{E}_{g}[\xi]$ and $\mathscr{E}_{g}\left[\xi / \mathscr{F}_{t}\right]$ are the classical mathematical expectation and conditional expectation respectively

$$
\mathscr{E}_{g}[\xi]=E \xi ; \quad \mathscr{E}_{g}\left[\xi / \mathscr{F}_{l}\right]=E\left[\xi / \mathscr{F}_{l}\right] .
$$

Furthermore, Peng proved that $\mathscr{E}_{g}[\cdot]$ and $\mathscr{E}_{g}\left[\cdot / \mathscr{F}_{t}\right]$ preserve respectively many properties of the classical mathematical expectations and conditional expectations except linearity (see Theorem A.2 and Theorem A.3 in Appendix). For that reason, Peng calls the general expectation and general conditional expectation $g$-expectation and conditional $g$-expectation respectively (see Peng $[24,25]$ ).

The following counter-example shows that a conditional $g$-expectation does not exist without the assumption (H4).

EXAMPLE 2. Suppose $\xi \in L^{2}(\Omega, \mathscr{F}, P)$, let $g(y, z, t)=\mathrm{e}^{-t}$. We choose $r=1$. Then there is no $\eta \in L^{2}\left(\Omega, \mathscr{F}_{1}, P\right)$ satisfying $(2.2)$.

In fact, if there exists such $\eta$ satisfying (2.2), that is,

$$
\mathscr{E}_{g}\left[\xi \mathbf{1}_{A}\right]=\mathscr{E}_{g}\left(\eta \mathbf{1}_{A}\right), \quad \forall A \in \mathscr{F}_{1}
$$

In particular, if we choose $A=\Omega$

$$
\mathscr{E}_{g}[\xi]=\mathscr{E}_{g}[\eta],
$$

then, by Definition $2.1, \mathscr{E}_{g}[\xi]$ and $\mathscr{E}_{g}[\eta]$ are the value of the solution $\left(y_{t}\right)$ of the following BSDE at time $t=0$ corresponding to $X=\xi$ and $X=\eta$, respectively

$$
y_{t}=X+\int_{t}^{\infty} \mathrm{e}^{-s} y_{s} d s-\int_{0}^{\infty} z_{s} d W_{s} .
$$

Solving the above linear BSDE (see Theorem A.0 in Appendix) we have

$$
y_{t}=E\left[X \mathrm{e}^{\int_{t}^{\infty} \mathrm{e}^{-s} d s} / \mathscr{F}_{t}\right],
$$

thus $\mathscr{E}_{g}[\xi]=\mathrm{e} E \xi$ and $\mathscr{E}_{g}[\eta]=\mathrm{e} E \eta$. That is,

$$
E \xi=E \eta
$$


On the other hand, since $\eta \mathbf{1}_{A}$ is $\mathscr{F}_{1}$ measurable, by Lemma $2.3, \mathscr{E}_{g}\left[\eta \mathbf{1}_{A}\right]$ is equal to the value of the solution $\left(\bar{y}_{t}\right)$ of the following BSDE at time $t=0$

$$
y_{t}=\eta+\int_{t}^{1} \mathrm{e}^{-s} y_{s} d s-\int_{0}^{1} z_{s} d W_{s}
$$

that is,

$$
\mathscr{E}_{g}[\eta]=\mathrm{e}^{1-\mathrm{e}^{-1}} E \eta
$$

Consequently, e $E \xi=\mathscr{E}_{g}[\xi]=\mathscr{E}_{g}[\eta]=\mathrm{e}^{1-\mathrm{e}^{-1}} E \eta$, which is contrary to (EQ).

For notational simplicity, in the rest of this paper we always assume that $g$ satisfies (H1)-(H4).

DEFINITION 2.5. A real value $\mathscr{F}_{t}$-adapted process $\left\{X_{t}\right\}_{t \geq 0}$ is called a $g$-martingale (supermartingale, submartingale) if for each $t \in[0, \infty), E\left|X_{t}\right|^{2}<\infty$, and for $0 \leq s \leq t<\infty$,

$$
\left.\mathscr{E}_{g}\left[X_{t} / \mathscr{F}_{s}\right]=X_{s} \quad \text { (respectively } \leq X_{s}, \geq X_{s}\right)
$$

The following lemma is from Chen and Peng [1, 2]. Let us outline the proof for the convenience of the reader.

LEMMA 2.6. Let $\left\{X_{t}\right\}$ be a g-supermartingale and $t_{0}, t_{1}, \ldots, t_{n}, \ldots$ be a strictly increasing sequence taking values in $[0, \infty)$. We denote by $U_{a}^{b}(X, n)$ the number of upcrossing interval $[a, b]$ of $\left\{X_{t_{i}}\right\}$ before $t_{n}$. Then

$$
\mathscr{E}_{-u}\left[U_{a}^{b}(X, n)\right] \leq \frac{1}{b-a} \mathscr{E}_{u}\left[\left(X_{i_{n}}-a\right)^{-}\right]
$$

PROOF. For $j=1,2, \cdots, n$, let $\left(y_{t}^{(j)}, z_{t}^{(j)}\right)$ be the solution of the following BSDE

$$
y_{t}^{(j)}=X_{t_{j}}+\int_{t}^{t_{j}} g\left(y_{s}^{(j)}, z_{s}^{(j)}, s\right) d s-\int_{t}^{t_{j}} z_{s}^{(j)} d W_{s}, \quad 0 \leq t \leq t_{j}
$$

Set

$$
a_{s}^{j}:= \begin{cases}\frac{g\left(y_{s}^{(j)}, z_{s}^{(j)}, s\right)}{z_{s}^{(j)}}, & \text { when } z_{s}^{(j)} \neq 0 \text { and } s \in\left(t_{j-1}, t_{j}\right] ; \quad a_{s}:=\sum_{j=1}^{n} a_{s}^{j} . \\ 0, & \text { otherwise, }\end{cases}
$$

By (H2) and (H4),

$$
|g(y, z, t)| \leq u(t)|z|, \quad \text { for }(y, z, t) \in R \times R^{d} \times R_{+} .
$$


Thus $\left|a_{t}\right| \leq u(t)$, for each $t>0$. Since, for each $j$,

$$
g\left(y_{s}^{(j)}, z_{s}^{(j)}, s\right)=a_{s} \cdot z_{s}^{(j)}, \quad s \in\left(t_{j-1}, t_{j}\right]
$$

BSDE (2.5) can be rewritten as

$$
y_{t}^{(j)}=X_{t_{j}}+\int_{t}^{t_{j}} a_{s} z_{s}^{(j)} d s-\int_{t}^{t_{j}} z_{s}^{(j)} d W_{s},=X_{t_{j}}-\int_{t}^{t_{j}} z_{s}^{(i)} d \bar{W}_{s}, \quad t \in\left[0, t_{j}\right]
$$

where $\bar{W}_{t}:=W_{t}-\int_{0}^{t} a_{s} d s$.

Let

$$
E\left[\frac{d Q}{d P} / \mathscr{F}_{t_{n}}\right]:=\exp \left\{-\frac{1}{2} \int_{0}^{t_{n}}\left|a_{s}\right|^{2} d s+\int_{0}^{t_{n}} a_{s} d W(s)\right\}
$$

By the Girsanov Theorem, $\left\{\bar{W}_{t}\right\}$ is a $Q$ - Brownian motion.

Since

$$
\begin{aligned}
& E \exp \left(-\frac{1}{2} \int_{0}^{t_{n}}\left(2 a_{s}\right)^{2} d s+\int_{0}^{t_{n}} 2 a_{s} d W_{s}\right)=1, \\
\left(E_{Q}\left|X_{t_{n}}\right|\right)^{2}= & \left(E\left|X_{t_{n}} \frac{d Q}{d P}\right|\right)^{2} \\
\leq & E X_{t_{n}}^{2} E \exp \left(-\frac{1}{2} \int_{0}^{t_{n}}\left(2 a_{s}\right)^{2} d s+\int_{0}^{t_{n}} 2 a_{s} d W_{s}\right) \exp \left(\int_{0}^{t_{n}} a_{s}^{2} d s\right) \\
\leq & E X_{t_{n}}^{2} \exp \left(\int_{0}^{t_{n}} u^{2}(s) d s\right)<\infty,
\end{aligned}
$$

which means that $\left\{X_{t_{n}}\right\}_{n \geq 0}$ is integrable under $Q$.

Taking conditional expectation $E_{Q}\left[\cdot / \mathscr{F}_{t}\right]$ on both sides of BSDE (2.8),

$$
\mathscr{E}_{g}\left[X_{t_{j}} / \mathscr{F}_{t}\right]=y_{t}^{(j)}=E_{Q}\left[X_{t_{j}} / \mathscr{F}_{t}\right]
$$

Since $X_{t}$ is a $g$-supermartingale,

$$
E_{Q}\left[X_{t_{j}} / \mathscr{F}_{t_{j-1}}\right]=\mathscr{E}_{g}\left[X_{t_{j}} / \mathscr{F}_{t_{j-1}}\right] \leq X_{t_{j-1}}, \quad j=1,2, \ldots, n .
$$

Thus $\left\{X_{t_{j}}\right\}_{i=1}^{n}$ is a $Q$-supermartingale with respect to $\left\{\mathscr{F}_{t_{i}}\right\}_{i=1}^{n}$.

Applying the classical upcrossing inequality of supermartingales, we have

$$
E_{Q}\left[\mathbf{U}_{a}^{b}(X, n)\right] \leq \frac{E_{Q}\left[\left(X_{t_{n}}-a\right)^{-}\right]}{b-a} .
$$

Note that, by Theorem A.3 (2)(ii) in Appendix, for $\xi \in L^{2}(\Omega, \mathscr{F}, P)$,

$$
\mathscr{E}_{-u}[\xi] \leq E_{Q}[\xi] \leq \mathscr{E}_{u}[\xi]
$$

This with (2.9) proves the result. 


\section{Convergence theorem of $g$-martingale}

From Theorem 1.3, we can obtain the following corollary.

COROLLARY 3.1. If $\left\{X_{t}\right\}$ is a g-martingale (non-negative g-submartingale), then there exists a constant $C$ such that

$$
E \sup _{t \geq 0}\left|X_{t}\right|^{2} \leq C \sup _{t \geq 0} E X_{t}^{2}
$$

PROOF. Suppose $\left\{X_{r}\right\}$ is a $g$-martingale, then for any $0 \leq t \leq T<\infty$, by the definition of $g$-martingale, there exists $z$ such that $(X, z)$ satisfies the BSDE

$$
X_{t}=X_{T}+\int_{t}^{T} g\left(X_{s}, z_{s}, s\right) d s-\int_{t}^{T} z_{s} d W_{s}
$$

By Theorem 1.3, there exists a constant $C$ such that $E \sup _{0 \leq t \leq T}\left|X_{t}\right|^{2} \leq C E\left|X_{T}\right|^{2} \leq$ $C \sup _{t \geq 0} E\left|X_{t}\right|^{2}$. From this, we obtain our result.

Using Lemma 2.6, we can easily prove the so-called convergence theorem of $g$-martingales.

THEOREM 3.2. If $\left\{X_{t}\right\}_{t \geq 0}$ is a g-martingale such that $\sup _{t \geq 0} E\left|X_{t}\right|^{2}<\infty$, then $X_{\infty}:=\lim _{t \rightarrow \infty} X_{t}$ exists almost surely and $X_{\infty} \in L^{2}(\Omega, \mathscr{F}, P)$. Furthermore, for any $t \in[0, \infty]$, we have

$$
\mathscr{E}_{g}\left[X_{\infty} / \mathscr{F}_{t}\right]=X_{t}, \quad \text { almost surely. }
$$

PROOF. Let $Q$ denote the set of rational number of $R$ and $a, b \in Q$ be such that $a<b$. Suppose that $\left\{t_{1}, \ldots, t_{n}, \ldots\right\}$ is a strictly increasing sequence with value in $R_{+}$and let $U_{a}^{b}(X, n)$ be the number of upcrossing interval $[a, b]$ of $\left\{X_{t_{i}}\right\}_{i=1}^{n}$ before $t_{n}$. Set $U_{a}^{b}(X):=\lim _{n \rightarrow \infty} U_{a}^{b}(X, n)$.

Since $\left\{\mathbf{1}_{\left\{U_{a}^{b}(X, n)>k\right\}}\right\}_{n \geq 0}$ is a bounded and increasing sequence, it follows by Theorem A.3(2)(iv), Lemma 2.6 and Theorem A.3(2)(iii) in Appendix that

$$
\begin{aligned}
\mathscr{E}_{-u}\left[\mathbf{1}_{\left\{U_{a}^{b}(X)>k\right\}}\right] & =\lim _{n \rightarrow \infty} \mathscr{E}_{-u}\left[\mathbf{1}_{\left\{U_{a}^{b}(X, n)>k\right\}}\right] \leq \lim _{n \rightarrow \infty} \mathscr{E}_{-u}\left[\frac{1}{k} U_{a}^{b}(X, n)\right] \\
& \leq \frac{1}{k(b-a)} \sup _{n \geq 0} \mathscr{E}_{u}\left[\left(X_{t_{n}}-a\right)^{-}\right] \leq \frac{1}{k(b-a)}\left(a^{+}+\sup _{n \geq 0} \mathscr{E}_{u}\left[X_{t_{n}}^{-}\right]\right) \\
& \leq \frac{1}{k(b-a)}\left(a^{+}+C \sup _{n \geq 0} E\left|X_{t_{n}}\right|^{2}\right),
\end{aligned}
$$


where $C$ is a constant defined in Theorem A.3(2)(iii) in Appendix. Let $k \rightarrow \infty$, then

$$
\mathscr{E}_{-u}\left[\mathbf{1}_{\left(\mathrm{U}_{a}^{b}(X)=\infty\right)}\right] \leq \lim _{k \rightarrow \infty} \frac{1}{k(b-a)}\left(a^{+}+C \sup _{n \geq 0} E\left|X_{t_{n}}\right|^{2}\right)=0 .
$$

Thus

$$
P\left(\left\{\mathbf{U}_{a}^{b}(X)=\infty\right\}\right)=0, \quad \text { for } a<b
$$

Finally, we obtain

$$
P\left(\bigcup_{\substack{a<b \\ a, b \in \mathbf{Q}}}\left\{\omega: \mathbf{U}_{a}^{b}(X)(\omega)=\infty\right\}\right)=0
$$

But

$$
\left\{\omega: \liminf _{n \rightarrow \infty} X_{t_{n}}(\omega)<\varlimsup_{n \rightarrow \infty} X_{t_{n}}(\omega)\right\}=\bigcup_{\substack{a<b \\ a, b \in Q}}\left\{\omega: \mathbf{U}_{a}^{b}(X)(\omega)=\infty\right\}
$$

and it follows that

$$
P\left(\omega: \liminf _{n \rightarrow \infty} X_{t_{n}}(\omega)<\varlimsup_{n \rightarrow \infty} X_{t_{n}}(\omega)\right)=0 .
$$

Let $H:=\left\{\omega: \liminf _{n \rightarrow \infty} X_{t_{n}}(\omega) \neq \varlimsup_{\lim _{n \rightarrow \infty}} X_{t_{n}}(\omega)\right\}$, then $P(H)=0$. Thus the limit of $\left\{X_{t_{n}}\right\}$ exists almost surely.

Set

$$
X_{\infty}:= \begin{cases}\lim _{n \rightarrow \infty} X_{t_{n}}(\omega), & \omega \notin H \\ 0, & \omega \in H\end{cases}
$$

then $\lim _{n \rightarrow \infty} X_{t_{n}}=X_{\infty}$ almost surely.

It then follows from Corollary 3.1 and the dominated convergence theorem that $\left\{X_{t_{n}}\right\} \rightarrow X_{\infty}$ in $L^{2}(\Omega, \mathscr{F}, P)$.

Now we prove (3.1). Since $\left\{X_{t}\right\}$ is $g$-martingale,

$$
\mathscr{E}_{g}\left[X_{t_{j}} / \mathscr{F}_{t}\right]=X_{t}, \quad \text { for } t_{j}>t
$$

By the definition of $\mathscr{E}_{g}[\cdot],\left\{X_{t}\right\}$ is the solution of BSDE:

$$
X_{t}=X_{t_{j}}+\int_{t}^{\infty} g\left(X_{s}, z_{s}, s\right) d s-\int_{t}^{\infty} z_{s} d W_{s}
$$

Note that $E\left|X_{t_{j}}-X_{\infty}\right|^{2} \rightarrow 0$ as $j \rightarrow \infty$. Taking the limit on both sides of BSDE (3.2) and applying Theorem 1.4, we complete the proof. 


\section{Some applications in security markets and economic theory}

A. Application in the pricing of contingent claims in incomplete markets It is well known that, in incomplete security markets, for a given contingent claim $\xi$, the maximum price of the contingent claim at time $t$ is given by

$$
V_{t}=\underset{v \in D}{\operatorname{ess} \sup } E_{Q^{v}}\left[\xi / \mathscr{F}_{t}\right]
$$

where

(1) $D$ is the set of all $R^{d}$-valued $\mathscr{F}_{t}$-adapted processes $\left\{v_{t}\right\}$ such that for any $T>0$, $E \int_{0}^{T}\left|v_{s}\right|^{2} d s<\infty$

(2) for any $v \in D, Q^{v}$ is a probability measure such that

$$
E\left[\frac{d Q^{v}}{d P} / \mathscr{F}_{T}\right]:=\exp \left(-\frac{1}{2} \int_{0}^{T}\left|v_{s}\right|^{2} d s+\int_{0}^{T} v_{s} d \bar{W}_{s}\right)
$$

(3) $\left\{\bar{W}_{t}\right\}$ is a semimartingale such that for each $v \in D,\left\{\bar{W}_{t}\right\}$ is a $Q^{v}$-martingale.

For the ease of exposition, in this section we assume that for any $v \in D,\left\{\bar{W}_{\imath}\right\}$ is a $Q^{v}$ Brownian motion.

El Karoui and Quenez [9] and Kramkov [17] showed that under the assumption $\xi \geq 0$ and $\sup _{v \in D} E_{Q^{v}} \xi<\infty$ for each $v \in D\left\{V_{t}\right\}$ is a $Q^{v}$-supermartingale and has the following optional decomposition theorem:

$$
V_{t}=V_{0}+\int_{0}^{t} H_{s} d \bar{W}_{s}-A_{t}
$$

where $\left\{H_{t}\right\}$ is a predictable process and $\left\{A_{t}\right\}$ is an optional increasing process. Furthermore, Kramkov gives a counter-example to show that $V_{\infty}:=\lim _{t \rightarrow \infty} V_{t} \neq \xi$.

We naturally present the following problems:

(1) Under which conditions is the limit of $\left\{V_{t}\right\}$ equal to $\xi$ ?

(2) How to calculate $\left\{H_{t}\right\}$ and $\left\{A_{t}\right\}$ in optional decomposition theorem (4.2)?

(3) If $\xi$ is not positive, does the above decomposition theorem still hold?

Let us denote by $D_{1}:=\{V: V \in D$ such that $|V(t)| \leq u(t)$, for $t \geq 0\}$, where $u$ is defined in (H2) and (H3). In this subsection, we show

THEOREM 4.1. If we replace $D$ in (4.1) by $D_{1}$ and assume that $\xi \in L^{2}(\Omega, \mathscr{F}, P)$ and $\left\{V_{t}\right\}$ is the process defined in (4.1), then

(1) $\left\{V_{t}\right\}$ is a g-martingale (where $\left.g(y, z, t):=u(t)|z|\right)$, thus $X_{\infty}:=\lim _{t \rightarrow \infty} V_{t}$ exists almost surely and $X_{\infty}=\xi$; 
(2) the predictable process $\left\{H_{t}\right\}$ and optional increasing process $\left\{A_{t}\right\}$ in (4.2) are given by: $H_{t}=z_{t}$ and $A_{t}=\int_{0}^{t} u(s)\left|H_{s}\right| d s$. Here $\left\{z_{t}\right\}$ is the solution of the following infinite time interval BSDE:

$$
y_{t}=\xi+\int_{t}^{\infty} u(s)\left|z_{s}\right| d s-\int_{t}^{\infty} z_{s} d \bar{W}_{s} .
$$

PROOF. By Theorem 1.2, let $(y, z)$ be the solution of BSDE (4.3). We only need to prove that $\left\{V_{t}\right\}$ defined in (4.1) is the solution of BSDE (4.3). In fact, for any $v \in D$, set $y_{t}^{v}:=E_{Q^{v}}\left[\xi / \mathscr{F}_{t}\right]$. By Bayes' rule, $\left\{y^{v}\right\}$ solves the following BSDE:

$$
y_{t}^{v}=\xi+\int_{t}^{\infty} v_{s} z_{s} d s-\int_{t}^{\infty} z_{s} d \bar{W}_{s}
$$

Note that $v_{t} z \leq u(t)|z|$ for all $t>0$ and any $z \in R^{d}$. By the Comparison Theorem (Theorem A.2 in Appendix), we have $y_{t}^{v} \leq y_{t}$ for all $t>0$. Thus

$$
V_{t}=\underset{v \in D_{1}}{\operatorname{esssup}} y_{t}^{v} \leq y_{t}, \quad t>0 .
$$

On the other hand, for the solution $(y, z)$ of $\operatorname{BSDE}(4.3)$, set

$$
a_{t}= \begin{cases}\frac{u(t)\left|z_{t}\right|}{z_{t}}, & z_{t} \neq 0 \\ 0, & z_{t}=0 .\end{cases}
$$

Obviously, $a:=\left\{a_{t}\right\} \in D_{1}$ and BSDE (4.3) can be rewritten as the following linear BSDE:

$$
y_{t}=\xi+\int_{t}^{\infty} a_{s} z_{s} d s-\int_{t}^{\infty} z_{s} d \bar{W}_{s} .
$$

Solving the above linear BSDE (see Theorem A.0 in Appendix), we have

$$
y_{t}=E_{Q^{a}}\left[\xi / \mathscr{F}_{t}\right]
$$

It then follows, since $\left\{a_{t}\right\} \in D_{1}$, that

$$
y_{t}=E_{Q^{[}}\left[\xi / \mathscr{F}_{t}\right] \leq \underset{v \in D_{1}}{\operatorname{ess} \sup } E_{Q^{v}}\left[\xi / \mathscr{F}_{t}\right]=V_{t}
$$

Consequently, from (4.4) and (4.5), we have $y_{t}=V_{t}$, for all $t \geq 0$.

Furthermore, for any $T>t>0$, from BSDE (4.3),

$$
V_{t}=V_{T}+\int_{t}^{T} u(s)\left|z_{s}\right| d s-\int_{t}^{T} z_{s} d \bar{W}_{s}
$$


which means that $\left\{V_{t}\right\}$ is a $g$-martingale (where $\left.g(y, z, t)=u(t)|z|\right)$. By Theorem 3.2, $V_{\infty}:=\lim _{t \rightarrow \infty} V_{t}$ exists. Obviously, $V_{\infty}=\xi$. The proof of (1) is complete.

Since $\left\{V_{r}\right\}$ is the solution of BSDE (4.3), BSDE (4.3) can be rewritten as

$$
V_{t}=V_{0}+\int_{0}^{t} z_{s} d \bar{W}_{s}-\int_{0}^{t} u(s)\left|z_{s}\right| d s
$$

Set $A_{t}:=\int_{0}^{t} u(s)\left|z_{s}\right| d s$ and $H_{t}:=z_{t}$, the proof of (2) is complete.

B. Application in economic theory It is interesting that the notions of $g$-expectations can be applied in economic theory.

As we know, in the theory of individual behaviour toward uncertainty, Savage proposed axioms regarding a binary relation on the set of acts which are necessary and sufficient for it to be representable by the expectation of a von Neumann-Morgenstern utility function. However, the famous Ellsberg and Allais Paradox have demonstrated that the Savage axioms under uncertainty are contradicted. Such an evidence has stimulated the development of generalized models of preference designed to accommodate aversion to ambiguity or uncertainty. The generalization that we consider now is the Choquet expected utility, in which the subjective probability measure of Savage is replaced by a non-additive probability that is more precisely termed a capacity (see Epstein [10], Epstein and Wang [11], Gilboa and Schmeidler [13], Schmeidler [26]). The definition of $g$-expectation in this paper is related closely to this problem.

In fact, for any $A \in \mathscr{F}$, let us denote $P_{g}(A)$ by

$$
P_{g}(A):=\mathscr{E}_{g}\left(\mathbf{1}_{A}\right) .
$$

Then it is easy to check that $P_{g}(\cdot)$ is a Choquet capacity on $(\Omega, \mathscr{F})$, that is,

(i) $P_{g}(\Omega)=1, P_{g}(\emptyset)=0$;

(ii) assume $A, B \in \mathscr{F}$, if $A \subset B$, then $P_{g}(A) \leq P_{g}(B)$;

(iii) assume $A_{n}(n=1,2, \ldots), A \in \mathscr{F}$, if $A_{n} \downarrow A\left(A_{n} \uparrow A\right)$, then $P_{g}\left(A_{n}\right) \downarrow P_{g}(A)$ $\left(P_{g}\left(A_{n}\right) \uparrow P_{g}(A)\right)$, as $n \rightarrow \infty$.

PROOF. (i) is trivial; (ii) follows directly from Theorem A.3(2)(i) in Appendix; (iii) follows from Theorem 1.3.

The following is the definition of uncertainty aversion given by Schmeidler [26]. More recently, Epstein [10] gave an abstract definition of an uncertainty aversion.

DEFINITION. A binary relation $\succeq$ is said to reveal uncertainty aversion, if for any act $\xi, \eta$ and $X$, and for any $\alpha$ in $[0,1]:$ if $\xi \succeq X$ and $\eta \succeq X$, then

$$
\alpha \xi+(1-\alpha) \eta \geq X \text {. }
$$


For definition of a strict uncertainty aversion, the conclusion should be a strict preference $\succ$.

By the definition of uncertainty aversion, we can obtain

THEOREM 4.2. If $g(y, z, t):=-u(t)|z|$ and $\xi, \eta \in L^{2}(\Omega, \mathscr{F}, P)$, then the following relation $\succeq$ denoted by

$$
\xi \succeq \eta \Longleftrightarrow \mathscr{E}_{-u}[\xi] \succeq \mathscr{E}_{-u}[\eta]
$$

is an uncertainty aversion.

The next result follows from the next lemma.

LEMMA 4.3. Suppose $\xi, \eta \in L^{2}(\Omega, \mathscr{F}, P)$, then for all $\alpha \in[0,1]$

$$
\mathscr{E}_{-u}[(1-\alpha) \xi+\alpha \eta] \geq(1-\alpha) \mathscr{E}_{-u}[\xi]+\alpha \mathscr{E}_{-u}[\eta] .
$$

PROOF. For any $X \in L^{2}(\Omega, \mathscr{F}, P)$, let $\left(y^{X}, z^{X}\right)$ be the solution of BSDE:

$$
y_{t}=X-\int_{t}^{\infty} u(s)\left|z_{s}\right| d s-\int_{t}^{\infty} z_{s} d W_{s} .
$$

It is easy to check that $\left((1-\alpha) y_{t}^{\xi}+\alpha y_{t}^{\eta},(1-\alpha) z_{t}^{\xi}+\alpha z_{t}^{\eta}\right)$ is the solution of BSDE:

$$
y_{t}=(1-\alpha) \xi+\alpha \eta-\int_{t}^{\infty} u(s)\left[\left|z_{s}\right|-f(s)\right] d s-\int_{t}^{\infty} z_{s} d W_{s},
$$

where $f(t):=(1-\alpha)\left|z_{t}^{\xi}\right|+\alpha\left|z_{t}^{\eta}\right|-\left|(1-\alpha) z_{t}^{\xi}+\alpha z_{t}^{\eta}\right| \geq 0$. By the Comparison Theorem (see Theorem A.2 in Appendix), we have

$$
y_{t}^{(1-\alpha) \xi+\alpha \eta} \geq(1-\alpha) y_{t}^{\xi}+\alpha y_{t}^{\eta} .
$$

In particular, when $t=0$, by the definition of $\mathscr{E}_{g}[\cdot]$, we have $\mathscr{E}_{-u}[(1-\alpha) \xi+\alpha \eta] \geq$ $(1-\alpha) \mathscr{E}_{-u}[\xi]+\alpha \mathscr{E}_{-u}[\eta]$.

ProOF OF THEOREM 4.2. Since

$$
\mathscr{E}_{-u}[(1-\alpha) \xi+\alpha \eta] \geq(1-\alpha) \mathscr{E}_{-u}[\xi]+\alpha \mathscr{E}_{-u}[\eta] \geq X
$$

we obtain the result. 


\section{Appendix}

The following are some theorems used in this paper.

THEOREM A.0. Suppose $\xi \in L^{2}(\Omega, \mathscr{F}, P),\left\{a_{t}\right\}$ and $\left\{b_{t}\right\}$ are two adapted processes such that for any $t \geq 0,\left|a_{t}\right| \leq v(t)$ and $\left|b_{t}\right| \leq u(t)$. Let $\{f(t)\}$ be an adapted process such that $E\left(\int_{0}^{\infty}|f(s)| d s\right)^{2}<\infty$ (where $v$ and $u$ are Lipschitz constants of $g$ in $(\mathrm{H} 2)$ and $(\mathrm{H} 3))$. Let $(y, z)$ be the solution of the following linear BSDE:

$$
y_{t}=\xi+\int_{t}^{\infty}\left[a_{s} y_{s}+b_{s} z_{s}+f(s)\right] d s-\int_{t}^{\infty} z_{s} d W_{s} .
$$

Then there exists a probability measure $Q$ such that

$$
y_{t}=E_{Q}\left[\xi e^{\int_{t}^{\infty} a_{s} d s}+\int_{t}^{\infty} f(s) e^{\int_{t}^{s} a_{r} d r} d s / \mathscr{F}_{t}\right]
$$

where

$$
E\left[\frac{d Q}{d P} / \mathscr{F}_{t}\right]:=\exp \left(-\frac{1}{2} \int_{0}^{t} b_{s}^{2} d s+\int_{0}^{t} b_{s} d W_{s}\right), \quad t \geq 0 .
$$

ProOF. This result can be proved in much the same way as in Karoui, Peng and Quenez [8], Peng [22, 23, 25]. Set

$$
Q_{t}:=\exp \left(\int_{0}^{t} b_{s} d W_{s}-\frac{1}{2} \int_{0}^{t}\left|b_{s}\right|^{2} d s\right) .
$$

Applying Itô's formula to $Q_{t} y_{t} \exp \left(\int_{0}^{t} a_{s} d s\right)$, we have the result.

The following lemma plays an important role in the proof of Theorem A.2, Theorem A.3 and Theorem A.4.

Lemma A.1. Suppose $\xi_{1}, \xi_{2} \in L^{2}(\Omega, \mathscr{F}, P)$ and $(\mathrm{H} 1)$, (H2) and (H3) hold for $g_{i}$ $(i=1,2)$, let $\left(y_{i}^{i}, z_{1}^{i}\right)$ be the solutions of the BSDE (1.1) corresponding to $g=g_{1}$, $g=g_{2}$ and $\xi=\xi_{i}, \xi=\xi_{2}$, respectively. Then there exist a probability measure $Q$ and an adapted process $\left\{a_{t}\right\}$ which satisfies for any $t \geq 0,\left|a_{t}\right| \leq v(t)$ such that

$$
y_{t}^{1}-y_{t}^{2}=E_{Q}\left[\left(\xi_{1}-\xi\right) e^{\int_{0}^{\infty} a_{s} d s}+\int_{t}^{\infty}\left(g_{1}\left(y_{s}^{2}, z_{s}^{2}, s\right)-g_{2}\left(y_{s}^{2}, z_{s}^{2}, s\right)\right) e^{\int_{t}^{s} a_{r} d r} d s / \mathscr{F}_{t}\right] \text {. }
$$

PROOF. Without loss of generality, we assume that the Brownian motion in BSDE (1.1) is 1-dimensional, that is, $d=1$. Set

$$
a_{s}:= \begin{cases}\frac{g_{1}\left(y_{s}^{1}, z_{s}^{1}\right)-g_{1}\left(y_{s}^{2}, z_{s}^{1}, s\right)}{y_{s}^{1}-y_{s}^{2}}, & \text { if } y_{s}^{1} \neq y_{s}^{2}, \\ 0, & \text { otherwise, }\end{cases}
$$




$$
b_{s}:= \begin{cases}\frac{g_{1}\left(y_{s}^{2}, z_{s}^{1}\right)-g_{1}\left(y_{s}^{2}, z_{s}^{2}\right)}{z_{s}^{1}-z_{s}^{2}}, & \text { if } z_{s}^{1} \neq z_{s}^{2} \\ 0, & \text { otherwise }\end{cases}
$$

Thus, $E \int_{0}^{\infty}\left|a_{s}\right| d s \leq \int_{0}^{\infty} v(s) d s<\infty$ and $E \int_{0}^{\infty}\left|b_{s}\right|^{2} d s \leq \int_{0}^{\infty} u^{2}(s) d s<\infty$ since the assumption (H2) and (H3) hold on $g$, where $v$ and $u$ are the Lipschitzian constants of $g$ in (H2) and (H3). In the above notations, the following BSDE

$$
y_{t}^{1}-y_{t}^{2}=\xi_{1}-\xi_{2}+\int_{t}^{\infty}\left(g_{1}\left(y_{s}^{1}, z_{s}^{1}, s\right)-g_{2}\left(y_{s}^{2}, z_{s}^{2}, s\right) d s\right) d s-\int_{t}^{\infty}\left(z_{s}^{1}-z_{s}^{2}\right) d W_{s}
$$

can be rewritten as

$$
\begin{aligned}
y_{t}^{1}-y_{t}^{2}= & \xi_{1}-\xi_{2}+\int_{t}^{\infty}\left[a_{s}\left(y_{s}^{1}-y_{s}^{2}\right)+b_{s}\left(z_{s}^{1}-z_{s}^{2}\right)+\left(g_{1}\left(y_{s}^{2}, z_{s}^{2}, s\right)-g_{2}\left(y_{s}^{2}, z_{s}^{2}, s\right)\right)\right] d s \\
& -\int_{t}^{\infty}\left(z_{s}^{1}-z_{s}^{2}\right) d W_{s} .
\end{aligned}
$$

It then follows from Theorem A.0 that there exists a probability measure $Q$ such that $y_{t}^{1}-y_{t}^{2}=E_{Q}\left[\left(\xi_{1}-\xi_{2}\right) \mathrm{e}^{\int_{t}^{\infty} a_{s} d s}+\int_{t}^{\infty}\left(g_{1}\left(y_{s}^{2}, z_{s}^{2}, s\right)-g_{2}\left(y_{s}^{2}, z_{s}^{2}, s\right)\right) \mathrm{e}^{\int_{t}^{s} a_{r} d r} d s / \mathscr{F}_{t}\right]$, where

$$
E\left[\frac{d Q}{d P} / \mathscr{F}_{t}\right]:=\exp \left\{-\frac{1}{2} \int_{0}^{t} b_{s}^{2} d s+\int_{0}^{t} b_{s} d W_{s}\right\}, \quad t \geq 0
$$

Using Lemma A.1, we obtain the following Comparison Theorem.

THEOREM A.2. Under the assumptions of Lemma A.1, if $g_{1}(y, z, t) \geq g_{2}(y, z . t)$, for $(y, z, t) \in R \times R^{d} \times R_{+}$and $\xi_{1} \geq \xi_{2}$, we have:

(1) (Comparison Theorem.)

$$
y_{t}^{1} \geq y_{t}^{2}, \quad \text { for } t \geq 0 .
$$

(2) (Strict Comparison Theorem.) Furthermore, if $P\left(\xi_{1}>\xi_{2}\right)>0$, then

$$
y_{t}^{1}>y_{t}^{2}, \quad \text { for } t \in[0, \infty) \text {. }
$$

Proof. (1) is obvious. We now prove (2). By Lemma A.1 and the assumption of the theorem, there exist a probability measure $Q$ and an adapted process $\left\{a_{t}\right\}$ which satisfies $\left|a_{t}\right| \leq v(t)$, for every $t \geq 0$, such that

$$
y_{t}^{1}-y_{t}^{2} \geq E_{Q}\left[\left(\xi_{1}-\xi\right) \mathrm{e}^{\int_{0}^{\infty} a_{s} d s} / \mathscr{F}_{t}\right] .
$$


Thus we have

$$
y_{t}^{1}-y_{t}^{2} \geq \mathrm{e}^{-\int_{t}^{\infty} v(s) d s} E_{Q}\left[\left(\xi_{1}-\xi_{2}\right) / \mathscr{F}_{t}\right] \geq \mathrm{e}^{-\int_{t}^{\infty} v(s) d s} E_{Q}\left[\left(\xi_{1}-\xi_{2}\right) \mathbf{1}_{\left[\xi_{1}>\xi_{2}\right]} / \mathscr{F}_{t}\right]>0 .
$$

The following basic properties of $g$-expectations and conditional $g$-expectations presented by Peng [25] are still true.

ThEOREM A.3. (1) Constant preserved: For each constant $c \in \mathbb{R}, \mathscr{E}_{g}[c]=c$.

(2) Monotonicity:

(i) If $\xi_{1}, \xi_{2} \in L^{2}(\Omega, \mathscr{F}, P)$ and $\xi_{1} \geq \xi_{2}$ almost surely, then $\mathscr{E}_{g}\left[\xi_{1}\right] \geq \mathscr{E}_{g}\left[\xi_{2}\right]$. Particularly, if $P\left(\xi_{1}>\xi_{2}\right)$, then $\mathscr{E}_{g}\left(\xi_{1}\right)>\mathscr{E}_{g}\left(\xi_{2}\right)$.

(ii) Let $\xi \in L^{2}(\Omega, \mathscr{F}, P)$, if $g_{1}(y, z, \cdot) \leq g_{2}(y, z, \cdot)$ for all $(y, z) \in \mathbb{R} \times \mathbb{R}^{d}$, then $\mathscr{E}_{g_{1}}[\xi] \leq \mathscr{E}_{g_{2}}[\xi]$. Particularly, $\mathscr{E}_{-\mu}[\xi] \leq \mathscr{E}_{g}[\xi] \leq \mathscr{E}_{\mu}[\xi]$.

(3) If $\xi \in L^{2}(\Omega, \mathscr{F}, P)$, then there exists a constant $C>0$ such that $\left(\mathscr{E}_{g}[|\xi|]\right)^{2} \leq$ $C E|\xi|^{2}$.

(4) Continuity: If $\xi_{n}, \xi \in L^{2}(\Omega, \mathscr{F}, P)$ and $E\left|\xi_{n}-\xi\right|^{2} \rightarrow 0$ as $n \rightarrow \infty$, then $\mathscr{E}_{g}\left(\xi_{n}\right) \rightarrow \mathscr{E}_{g}(\xi)$

PROOF. (1) follows from Lemma 2.3; (2) follows from Lemma A.1 and Theorem A.2; (3) and (4) follow from Theorem 3.1 and Theorem 1.3.

Similarly, we have

THEOREM A.4. (i) If $\xi$ is $\mathscr{F}_{t}$-measurable, $t \in[0, \infty)$, then $\mathscr{E}_{g}\left[\xi \mid \mathscr{F}_{t}\right] \equiv \xi$.

(ii) Associativity Property: For each $t, r \in[0, \infty)$, then $\mathscr{E}_{g}\left[\mathscr{E}_{g}\left[\xi \mid \mathscr{F}_{t}\right] \mid \mathscr{F}_{r}\right]=$ $\mathscr{E}_{g}\left[\xi \mid \mathscr{F}_{1 \wedge r}\right]$. Particularly, $\mathscr{E}_{g}\left[\mathscr{E}\left[\xi / \mathscr{F}_{t}\right]\right]=\mathscr{E}_{g}[\xi]$.

(iii) If $\xi_{1} \geq \xi_{2}$, then $\mathscr{E}_{g}\left[\xi_{1} \mid \mathscr{F}_{t}\right] \geq \mathscr{E}_{g}\left[\xi_{2} \mid \mathscr{F}_{t}\right]$.

(iv) For all $\xi \in L^{2}(\Omega, \mathscr{F}, P), \mathscr{E}_{-\mu}\left[\xi \mid \mathscr{F}_{t}\right] \leq \mathscr{E}_{g}\left[\xi \mid \mathscr{F}_{t}\right] \leq \mathscr{E}_{\mu}\left[\xi \mid \mathscr{F}_{t}\right], t \in[0, \infty)$.

\section{References}

[1] Z. Chen and S. Peng, 'Continuous properties of $g$-martingales', to appear in Chinese Ann. Math. (2000).

[2] - 'A general downcrossing inequality for g-martingales', Statist. Probab. Lett. 46 (2000), $169-175$.

[3] J. Cvitanic and I. Karatzas, 'Hedging contingent claims with constrained portfolios', Ann. Appl. Probab. 3 (1993), 652-681.

[4] J. Cvitanic and J. Ma, 'Hedging options for a large investor and forward-backward SDEs', to appear in Ann. Appl. Probab.. 
[5] R. Daling, 'Constructing of Gamma-martingales with prescribed limits, using BSDE', Ann. Probab. 23 (1995), 1234-1261.

[6] R. Daling and E. Pardoux, 'BSDE with random terminal time and applications to semilinear elliptic PDE', Ann. Probab. 3 (1997), 1135-1159.

[7] D. Duffie and L. Epstein, 'Stochastic differential utility', Econometrica 60 (1992), 353-394.

[8] N. El Karoui, S. Peng and M-C. Quenez, 'BSDEs in finance', Math. Finance 7 (1997), 1-71.

[9] N. El Karoui and M-C. Quenez, 'Dynamic programming and pricing of contingent claims in an incomplete markets', SIAM J. Control Optim. 33 (1995), 29-66.

[10] L. Epstein, 'A definition of uncertainty aversion', Rev. Econom. Studies 66 (1999), 579-608.

[11] L. Epstein and T. Wang, 'Intertemporal asset pricing under Knightain uncertainty', Econometrica 62 (1994), 283-322.

[12] - 'Uncertainty, risk-neutral measures and security price booms and crashes', J. Econom. Theory 67 (1995), 40-82.

[13] I. Gilboa and D. Schmeidler, 'Maxim expected utility with non-unique prior', J. Math. Econom. 18 (1989), 141-153.

[14] S. Hamadéne and J. P. Lepeltier, 'Zero-sum stochastic differential games and backward equations', System Control Lett. 24 (1995), 259-263.

[15] - 'Backward equations, stochastic control and zero-sum stochastic differential games', Stochastics 54 (1995), 221-231.

[16] Y. Hu and S. Peng, 'Solutions of backward-forward SDE', Probab. Theory Related Fields 103 (1995), 273-283.

[17] D. Kramkov, 'Optional decomposition of supermartingales and hedging contingent claims incomplete security markets', Probab. Theory Related Fields 105 (1996), 459-479.

[18] J. P. Lepeltier and J. S. Martin, 'BSDEs with continuous coefficients', Statist. Probab. Lett. 32 (1997), 425-430.

[19] E. Pardoux, 'Generalized discontinuous BSDEs', in: Pitman Res. Notes Math. Ser. 364 (1997), 207-219.

[20] E. Pardoux and S. Peng, 'Adapted solution of a backward stochastic differential equation', System Control Lett. 14 (1990), 55-61.

[21] E. Pardoux and S. Zhang, 'Generalized BSDEs and nonlinear Neumann boundary value problems', Probab. Theory Related Fields 110 (1998), 535-558.

[22] S. Peng, 'A general stochastic maximum principle for optimal control problems', SIAM J. Control Optim. 28 (1990), 966-979.

[23] - 'Probabilistic interpretation for systems of quasilinear parabolic partial differential equations', Stochastics Stochastics Rep. 37 (1991), 61-74.

[24] _ ' Monotonic limit theorem of BSDE and its application to Doob-Meyer decomposition theorem', Probab. Theory Related Fields 113 (1999), 473-499.

[25] - 'BSDE and related g-expectation', in: Pitman Res. Notes Math. Ser. 364 (1997), 141-159.

[26] D. Schmeidler, 'Subjective probability and expected utility without additivity', Econometrica 57 (1989), 571-587.

[27] L. Wasserman and J. Kadane, 'Bayes' theorem for Choquet capacities', Ann. Statist. 18 (1990), 1328-1339.

Department of Mathematics

Shandong University

Jinan

Shandong 250100

P. R. China 